\section{Verteilungsfragen und intergenerationelle Gerechtigkeit in der Ökologischen Ökonomie}

In einem frühen Versuch zu einer zusammenfassenden Darstellung des neuentstandenen Gebiets ecological economics hat R. Costanza (1991) diese Ökologische Ökonomie zutreffend als science and management of sustainability, als Lehre von der Nachhaltigkeit charakterisiert. Indem diese neuentstandene Disziplin sich mit guten Gründen auf das Konzept der Nachhaltigkeit (sustainability) zentriert, nimmt sie einen Begriff auf, der in gewisser Weise der ökonomischen Tradition seit Adam Smith (1723-90) und Vilfredo Pareto (1843-1923) diametral entgegensteht, weil er gerade die von jenen Ökonomen mit guten Gründen geforderte Trennung von Allokation und Distribution ebenso grundsätzlich in Frage stellt wie die Annahme unbeschränkten Wachstums im progressive state.

Es sollte kein Zweifel daran bestehen, daß die Konzentration auf Allokations- und Effizienzaspekte bei gleichzeitiger Ausblendung der Verteilungsproblematik eine wesentliche Voraussetzung für die Entwicklung ökonomischen Denkens war und insofern durchaus einen Beitrag zu wissenschaftlichem Fortschritt darstellt. Die Ökonomik, wie auch andere Einzelwissenschaften, entstand eben gerade dadurch, daß sie bewußt bestimmte Aspekte der Realität ausblendete und damit in einem reduzierten Modellrahmen zu disziplinärer Entwicklung und Erkenntnisfortschritt befähigt wurde. Es war wohl unvermeidlich, die mittelalterliche Einheit von Allokation und Distribution aufzubrechen, wie sie sich etwa im scholastischen Konzept des »pretium justum «, des gerechten Preises, manifestierte (vgl. auch Daly 1992, S. 109).

Stellt nun die erneute Einbeziehung von Verteilungsfragen in den ökonomischen Diskurs einen Rückfall in längst überwunden geglaubtes mittelalterliches Denken dar? Ich will im folgenden argumentieren, daß es sich hierbei um etwas grundsätzlich anderes handelt, nämlich um die Notwendigkeit, die ursprünglich sinnvolle, ja vielleicht sogar historisch notwendige Ausblendung von Verteilungsfragen nun auf einer höheren Stufe ökonomischer Reflexion wieder »aufzuheben«, und zwar im bekannten dreifachen Wortsinne Hegels. So wichtig aus innerwissenschaftlichen Gründen die vorläufige Suspension der Distributionsfrage aus dem ökonomischen Denken gewesen sein mag, sie ändert nichts daran, daß Verteilungsfragen ein wesentlicher Teil unserer sozialen Realität und des Bewußtseins der Menschen auch in entwickelten Marktgesellschaften sind. Wie tief verwurzelt trotz allem methodologischem Individualismus und aller Beschränkung auf Effizienzurteile Gerechtigkeitsfragen im Bewußtsein der Menschen bis heute sind, zeigt nicht zuletzt die umweltökonomischökologische Debatte, wenn etwa gegen den Einsatz ökologisch orientierter Steuern von seiten betroffener Industrien geltend gemacht wird, sie seien »ungerecht «, weil sie gerade jene Sektoren belasteten, die schon in der Vergangenheit große Anstrengungen zum Schutze der Umwelt unternommen hätten und die überdies in den vergangenen Jahrzehnten wesentliche Träger unseres wirtschaftlichen Wohlstandes gewesen sein.

Worum es also - vor allem in der Ökologischen Ökonomie - geht, ist die für jede Einzelwissenschaft immer wieder neu zu stellende Frage, ob und wie es ihr gelingt, zunächst in ihren Modellen ausgeblendete Teile der Realität auf »höherem Niveau « wieder in die disziplinäre, fachwissenschaftliche Untersuchung einzubeziehen. Die in den letzten zehn Jahren entstandene Ökologische Ökonomie läßt sich in der Tat auch am besten als ein Versuch verstehen, diese Wiedereinbeziehung der Distributionsproblematik aus ökologischer Perspektive nicht nur einzufordern, sondern selbst zu leisten, mindestens aber entscheidend voranzubringen. Da es aber im ökologischen Diskurs nicht um Verteilung als solche geht, sondern um das Distributionsproblem zwischen heutigen und künftigen Generationen einerseits und den Menschen des »reichen Nordens « und des »armen Südens « andererseits, muß auch ein dritter, bisher nicht erwähnter Gesichtspunkt zur Geltung kommen, nämlich der des Ausmaßes (scale) der Wirtschaft, das über den physischen Naturverbrauch entscheidet.

\section{Allokation, Distribution, Ausmaß}

Herman E. Daly (1992) hat eine ökologisch sinnvolle Differenzierung zwischen drei zentralen Zielen ökonomischen Handelns gemacht, indem er unterschied zwischen Allokation als relativer Aufteilung der Ressourcenströme auf alternative Verwendungsrichtungen (Güter und Dienstleistungen) auf der einen Seite und andererseits der Distribution als relativer Aufteilung der Ressourcenströme, wie sie sich in den produzierten Gütern und Dienstleistungen darstellen auf unterschiedliche (heute und künftig lebende) Menschen. Dieser wohlbekannten Zweiteilung fügt er nun noch eine dritte Komponente hinzu, nämlich das Ausmaß (scale), unter dem er den physischen Umfang des Durchsatzes versteht, den Strom von Materie und Energie aus der Umwelt als niedrigentropische Rohmaterialien und zurück zur Umwelt als hochentropische Abfälle. Man kann sich dieses Ausmaß als das Produkt aus Bevölkerung mal Ressourcenverbrauch pro Kopf vorstellen. Daly sieht das reale Bruttosozialprodukt als den vielleicht besten Indikator für das Ausmaß dieses Durchsatzes. Das Ausmaß der Wirtschaft als Zielgröße der Wirtschaftspolitik zielt auf die Erfassung - und unter dem Gesichtspunkt der Nachhaltigkeit auf die Begrenzung - des physischen Naturverbrauchs, wie er sich vor allem in der Energie- und Ressourcenintensität unseres Wirtschaftens widerspiegelt.

Während die mainstream economics durchaus die Existenz und die prinzipielle Berechtigung von Distributionsproblemen akzeptiert und diese nur aus vielerlei Gründen - vor allem aber wegen der angeblichen Unschärfe von Konzepten distributiver Gerechtigkeit - an den Rand ökonomischer Analyse gedrängt hat, ist ihr die Anerkenntnis vom Ausmaß des Wirtschaftens als selbständige Zielkategorie vollkommen fremd, ja strukturell zuwider: Für sie ist Ausmaß nichts anderes als ein allokatives Spezialproblem, das sich dann ergibt, wenn zuvor freie Naturgüter infolge wirtschaftlichen Wachstums knapp zu werden beginnen. Für traditionelle neoklassische Ökonomen ist das Problem »klar $\ll$ : Es geht nurmehr um die Bestimmung richtiger »Schattenpreise « für Naturgüter, und zwar möglichst in der Form, $\mathrm{da}$ sie als reale Preise auf möglichst perfekt funktionierenden Naturgutmärkten gebildet werden. Für sie ist die Knappheit von Naturgütern in keiner Weise unterscheidbar von der Knappheit produzíerter Güter, eben deswegen, weil die vielfältigen Leistungen der Natur nur als Inputs für Produktion und Konsum gesehen werden, nicht aber als eine eigenständige Lebensgrundlage menschlicher Existenz und damit als eine Produktionsvoraussetzung des Wirtschaftens schlechthin.

Genau an dieser Frage scheiden sich die Geister zwischen traditioneller und Ökologischer Ökonomie: Letztere betrachtet die Wirtschaft als einen - wenn auch sehr stark ausdifferenzierten und beängstigend angewachsenen - Teilbereich des Ökosystem; die mainstream economics sieht jedoch (wenn auch meist unausgesprochen) Natur primär als Inputlieferantin und damit das Ökosystem gerade umgekehrt als Teilbereich der Wirtschaft. Daly (1992) erkennt hier zu Recht einen grundlegenden Auffassungsunterschied, den er mit der Differenz zwischen ptolemäischem und kopernikanischem Weltbild vergleicht: So, wie nach Ptolemäus die Erde der Mittelpunkt des Universums war, ist für die mainstream economics die Wirtschaft das Zentrum aller menschlichen und nicht-menschlichen Aktivitäten. Genau aus diesem Grunde vermag sie auch keinen prinzipiellen Unterschied zwischen Natur- und Sachkapital zu erkennen und entscheidet sich, sofern sie sich überhaupt mit der Nachhaltigkeitsfrage befaßt, für ein Konzept schwacher Nachhaltigkeit (weak sustainability). Es kommt nach dieser Auffassung dann nicht mehr auf den Naturerhalt als solchen an, sondern allenfalls auf den Erhalt des aggregierten Wertes yon Natur- und Sachkapital, und zwar unabhängig von der Art seiner Zusammensetzung (vgl. Nutzinger, 1995, S. 249).

Konzepte wie das der schwachen Nachhaltigkeit lassen sich ideologiekritisch leicht denunzieren als ein Versuch, die Frage des Ausmaßes unter die Allokations- und Effizienzproblematik zu subsumieren und damit, um nochmals den Vergleich zu physikalischen Weltbildern heranzuziehen, als Versuch, durch Konstruktion immer neuer Epizyklen die Vorstellung gleichmäßiger Kreisbahnen zu retten. Indessen: Die ideologiekritische Aufdeckung der Schwächen neoklassischer Umwelt- und Naturbetrachtung löst noch keineswegs das konkrete Problem, wie nun Fragen der Verteilung innerhalb von Generationen 
und zwischen den Generationen unter dem $\mathrm{Ge}$ sichtspunkt des Naturerhalts positiv zu entscheiden sind.

\section{Inter- und intragenerationelle Verteilung sub specie naturae}

Nach einer vielzitierten Formel der Weltkommission für Umwelt und Entwicklung (WCED, 1987, S. 46) ist eine Entwicklung dann als nachhaltig zu bezeichnen, wenn sie »die Bedürfnisse der Gegenwart befriedigt, ohne zu riskieren, $\mathrm{da} ß$ künftige Generationen ihre eigenen Bedürfnisse nicht befriedigen können «. Die Attraktivität dieses Konzepts nachhaltiger Entwicklung ist offenkundig, denn dadurch wird es möglich, die durchaus divergierenden Vorstellungen und Interessen heutiger und künftig lebender Menschen wenigstens begrifflich auf einen Nenner zu bekommen; wie die Diskussionen der letzten acht Jahre gezeigt haben, war der Argwohn ökologischer Ökonomen nur zu berechtigt, daß hinter diesem Formelkompromiß die realen Interessendivergenzen verschleiert werden und eine allenfalls marginal korrigierte Wirtschaftspolitik des »Weiter so! mit dem Markenzeichen »nachhaltig « oder »dauerhaft-umweltgerecht « bemäntelt wird.

Tatsächlich ist die Weltkommission für Umwelt und Entwicklung dieser Gefahr stellenweise selbst erlegen; sie hat sich zwar zu dem Formelkompromiß der nachhaltigen Entwicklung durchgerungen, ihn aber inhaltlich so gefüllt, daß die Komponente der intergenerationellen Gerechtigkeit faktisch an den Rand gedrängt wurde. Das ist natürlich bei der zwangsläufig notwendigen $\mathrm{Be}$ setzung dieser Kommission mit heute lebenden Vertretern heutiger Generationen aus dem »reichen Norden « und dem »armen Süden « nicht verwunderlich. Zum einen werden, trotz gelegentlicher kritischer Andeutungen, die eindeutig nachhaltigkeitswidrigen Konsum- und Wachstumsmuster der industrialisierten .Volkswirtschaften zum Maßstab wirtschaftlicher Entwicklung gemacht, an dem sich auch die Länder der »Dritten Welt« bei ihren Bestrebungen um eine »nachholende Entwicklung« ausrichten, ohne daß deutliche Aussagen über die Notwendigkeit von Beschränkungen und Verzichten in den entwickelten Volkswirtschaften getroffen werden. Noch deutlicher wird diese Tendenz in der immer wieder aufscheinenden Gleichsetzung von nachhaltiger Entwicklung und nachhaltigem Wachstum, auf die insbesondere H. -J. Harborth (1991) hingewiesen hat. Besonders auffällig wird diese Äquivokation von sustainable development und sustainable growth in nahezu hymnischen Sätzen der Weltkommission wie den folgenden:

"Die Menschheit wäre durchaus in der Lage, die Voraussetzungen für eine dauerhafte Entwicklung zu schaffen; eine Entwicklung, die den gegenwärtigen Bedarf zu decken vermag, ohne gleichzeitig späteren Generationen die Möglichkeit zur Deckung des ihren zu verbauen. Zwar schließt eine solches Konzept eines dauerhaften Wachstums Grenzen ein - doch sind dies keine absoluten Grenzen. Es sind vielmehr lediglich technologische und gesellschaftliche Grenzen, die uns durch die Endlichkeit der Ressourcen und die begrenzte Fähigkeit der Biosphäre zum Verkraften menschlicher Einflußnahme gezogen sind. Technologische und gesellschaftliche Entwicklungen aber sind beherrschbar und können auf einen Stand gebracht werden, der eine neue Ära wirtschaftlichen Wachstums ermöglicht« (WCED 1987, S. 9f.).

Diese weitgehende Gleichsetzung von Wachstum. und Entwicklung ist natürlich unter Nachhaltigkeitsgesichtspunkten äußerst problematisch, denn es bedürfte schon heroischer Annahmen über die Grenzenlosigkeit und Ubiquität technischen Fortschritts, wenn man in einer biophysikalisch vielfach begrenzten Welt an der Schrankenlosigkeit wirtschaftlichen Wachstums festhalten wollte. Sie ist aber nicht nur Ausdruck alten ptolemäischen Denkens seit Adam Smith, das einen stationären Zustand der Wirtschaft nur als »trübe « auffassen kann.

Entscheidender ist die Tatsache, daß der Nachhaltigkeitsbegriff durch zwei starke distributive Komponenten charakterisiert ist, nämlich der Verteilungsgerechtigkeit innerhalb der heute lebenden Menschen und zwischen heutigen und künftigen Generationen, deren Einlösung unter stationären Bedingungen als aussichtsloser Kampf um einen gleichbleibenden oder gar schrumpfenden Kuchen erscheinen muß. Die optimistischen Annahmen der Weltkommission für Umwelt und Entwicklung und späterer Autoren über hohe Wachstumsraten unter den Bedingungen nachhaltiger Entwicklung kommen nicht von ungefähr: Will man aus naheliegenden politischen Gründen auf eine (schwer durchsetzbare) Umverteilung von Besitzständen »reicher Länder « zugunsten »ärmerer Länder« und heute lebender Menschen zugunsten künftiger Generationen verzichten und sich statt dessen auf die (eher durchsetzbare) Umverteilung von Bestandszuwächsen beschränken, dann bedarf es allerdings hoher Wachstumsraten, wenn man dem Ziel der Gerechtigkeit im Sinne einer Verminderung von Ungleichheit auch nur längerfristig nahekommen will. Verstärkt wird diese Wachstumsorientierung noch durch die dabei vertretene »engine of growth «-Theorie wirtschaftlicher Entwicklung, die weiterhin die Industrieländer als »Wachstumslokomotiven « der »Dritten Welt « sieht.

Genau hierin liegt die Crux von intra- und intergenerationeller Verteilungsgerechtigkeit im Konzept der nachhaltigen Entwicklung: Hohe Wachstumsraten lassen eine Umverteilung von Zuwächsen im Sinne von Gerechtigkeitszielen als politisch praktikabel erscheinen, und sie bedeuten keine Abkehr vom Pareto-Prinzip traditioneller Ökonomik, denn es kann dann »den Ärmeren « und »den künftigen Generationen « mehr gegeben werden, ohne daß von »den Reichen « und »den heute lebenden Menschen « etwas genommen werden müßte. Hohe Wachstumsraten, daran kann aber trotz aller begrüßenswerten und notwendigen Effizienzfortschritte in einzelnen Bereichen kein Zweifel bestehen, sind mit einem ernstgemeinten Konzept von Nachhaltigkeit im
Sinne des Naturerhalts unvereinbar, nicht zuletzt deswegen, weil in vielen Fällen durch mengenmäßige Zunahme des Ausmaßes per Saldo die Effizienzfortschritte pro Leistungseinheit mehr als aufgefressen werden. Das Drei-Liter-Auto, attraktives »Öko-Modell « industrialisierter Volkswirtschaften, wäre weltweit eine ökologische Katastrophe allerschlimmsten Ausmaßes.

Eines ist klar: Die Verknüpfung von Naturerhalt und inter- sowie intragenerationeller Verteilung im Konzept der nachhaltigen Entwicklung erleichtert die Aufgabe nicht. Soll aber nachhaltige Entwicklung nicht zum bloßen Schlagwort der 90er Jahre verkommen, so erfordert Nachhaltigkeit theoretisch und praktisch eine Abkehr vom traditionellen Wachstums- und Wohlstandsmodell und eine uns heutigen Menschen ganz ungewohnte und vielleicht unverständliche Bereitschaft zum Teilen und zum Verzichten. Aber ohne eine derartige Suffizienzrevolution, so schwierig und konfliktreich sie immer sein mag, wird langfristig weder Naturerhalt noch menschliches Überleben unter menschenwürdigen $\mathrm{Be}$ dingungen möglich sein.

Hans G. Nutzinger, Kassel

\section{Literatur}

- Binswanger, Mathias 1995: »Sustainable Development: Utopie in einer wachsenden Wirtschaft«, in: Zeitschrift für Umweltpolitik \& Umweltrecht, 18. Jg., S. 1-19

- Costanza, R. (Hg. ) 1991: »Ecological Economics. The Science and Management of Sustainability.« New York, Oxford

- Daly, Herman E. : »Allocation, distribution, and scale: towards an economics that is efficient, just and sustainable«, in: Ecological Economics, 6 (1992), S. 185-193

- Hampicke, Ulrich 1993: „Ökologische Ökonomie«. Opladen

- Hampicke, Ulrich 1995: „Ökologische Ökonomie«. In: M. Junkernheinrich u. a. (Hg. ): Handbuch zur Umweltökonomie. Berlin, S. 138-144

- Harborth, H. -J. 1991: »Dauerhafte Entwicklung statt globaler Selbstzerstörung. Eine Einführung in das Konzept des > sustainable development $\ll$. Berlin

- Lembke, Hans H.: »Umweltpolitik in der Nord-Süd-Dimension - UNCED 1992 und danach«, in: Zeitschrift für angewandte Umweltforschung, Jg. 5 (1992), S. 322-334

- Norton, Bryan G.: »Intergenerational equity and environmental decisions: a model using Rawls' veil of ignorance«, in: Ecological Economics, 1 (1989), S. 137-159

- Nutzinger, Hans G. 1995: »Nachhaltige Wirtschaftsweise und Energieversorgung. Konzepte, Bedingungen, Ansatzpunkte«. Marburg 1995

- Pearce, David 1987: »Economics, Equity and Sustainable Development«, in: Futures, Dezember 1988, S. 598-605

- WCED (Weltkommission für Umwelt und Entwicklung): »Unsere gemeinsame $\mathrm{Zu}$ kunft«. Greven 
(c) 20I0 Authors; licensee IÖW and oekom verlag. This is an article distributed under the terms of the Creative Commons Attribution Non-Commercial No Derivates License (http://creativecommons.org/licenses/by-nc-nd/3.o/), which permits unrestricted use, distribution, and reproduction in any medium, provided the original work is properly cited. 